J Am Acad Dermatol. 2009 January ; 60(1): 70-76. doi:10.1016/j.jaad.2008.06.032.

\title{
Effects of vulvodynia on quality of life
}

\author{
Maya Ponte, MD, $\mathrm{PhD}^{\mathrm{a}}$, Erika Klemperer, $\mathrm{MD}^{\mathrm{b}}$, Anju Sahay, $\mathrm{PhD}^{\mathrm{C}}$, and Mary-Margaret Chren, \\ MD \\ aSchool of Medicine, University of Californiae-San Francisco. \\ ${ }^{b}$ Department of Dermatology, University of Californiae-San Francisco. \\ 'Department of Medicine, University of Californiae-San Francisco.
}

\begin{abstract}
Background-The experiences of women with vulvodynia are poorly understood.

Objective-We sought to determine the effects of vulvodynia on quality of life.

Methods-We conducted a survey of 280 patients in a university-based vulvar disorders clinic. Skin-related quality of life was measured with a vulvar-specific version of Skindex-29.
\end{abstract}

Results-The response rate was 95\%; 101 patients (36\%) had vulvodynia, and 179 patients (64\%) had other vulvar conditions. Women with vulvodynia had significantly worse quality of life than patients with many other dermatologic conditions, and worse functioning than women with other vulvar conditions (mean functioning scores $[ \pm \mathrm{SD}]$ of patients with psoriasis, other vulvar conditions, and vulvodynia were $23 \pm 27,34 \pm 24$, and $44 \pm 22$, respectively, $P=.05$ ). A diagnosis of vulvodynia was the strongest independent correlate of poor quality of life (eg, for poor functioning, odds ratio $=1.8,95 \%$ confidence interval $1.0-3.1$ ).

Limitations-Limitations are single academic medical center and comorbid illnesses determined by self-report.

Conclusion-Vulvodynia has broad and substantial effects on quality of life.

Vulvodynia is a chronic pain disorder that is frequently distressing to the patient and challenging to the clinician. Although the terminology continues to evolve, the most recent definition proposed by the International Society for the Study of Vulvovaginal Disease (ISSVD) defines vulvodynia as vulvar discomfort, most often described as burning pain, occurring in the absence of relevant visible findings or a specific, clinically identifiable, neurologic disorder. ${ }^{1}$

The prevalence of vulvodynia is unclear, but the condition is probably more common than previously recognized. Some have estimated an incidence of up to $16 \%$ of women, ${ }^{2}$ suggesting that as many as 14 million women in the United States may experienced vulvodynia. Despite the significant number of women affected by this disorder, many aspects of vulvodynia remain poorly understood.

(C) 2008 by the American Academy of Dermatology, Inc.

Reprint requests: Mary-Margaret Chren, MD, San Francisco Veterans Affairs Medical Center, 4150 Clement St, 151R, San Francisco, CA 94121. E-mail: E-mail: chrenm@ derm.ucsf.edu.

Conflicts of interest: None declared.

Presented in part at the Annual Meeting of the American DermatoEpidemiology Network in Los Angeles, CA, on May 12, 2007. 
Our goal was to understand the impact of vulvodynia on the quality of life of affected women. We hypothesized that the quality-of-life effects of vulvodynia would be substantial compared with those of skin disorders and other vulvar conditions.

\section{METHODS}

Sample

Consecutive patients who attended our vulvar disorders clinic in selected months of 1998, 1999, and 2000 were invited to participate in this cross-sectional study by completing data forms before their appointments. Patients were referred to the clinic by their health care provider or were self-referred. All patients were seen in the clinic by one of the authors (E. $\mathrm{K}$.), who also made the final vulvar diagnosis. The study was approved by our institutional review board (protocol 06029061, renewal approved May 16, 2006).

\section{Data}

Skin-related quality of life was measured with Skindex-29. ${ }^{3}$ This validated instrument consists of 29 items in 3 subscales: symptoms, emotions, and functioning. In the symptoms subscale, items assess the frequency of physical experiences such as pain, itching, or irritation. Items in the emotions subscale inquire about emotional effects of a condition, including frustration, anger, and embarrassment. Items in the functioning subscale inquire about the effects of a condition on the social and physical functioning of a patient. Skindex-29 items inquire about quality of life during the prior 4 weeks. Scores are standardized from 0 (no effect) to 100 (maximal effect), and higher scores indicate worse quality of life. For this study, Skindex-29 items were adapted to inquire about the effects of the vulvar condition.

Patients also responded to additional items about demographic characteristics, health status, and other information pertaining to the vulvar condition or its quality-of-life effects that were not addressed in the generic Skindex instrument. Patients also responded "yes" or "no" to items inquiring about comorbid conditions such as frequent yeast infections, interstitial cystitis, depression, or anxiety disorder.

Medical records were reviewed to establish the final vulvar diagnosis. Participants were divided into two diagnostic categories: vulvodynia or other vulvar conditions. We used the ISSVD definition of vulvodynia ${ }^{1}$ : vulvar discomfort occurring in the absence of relevant visible findings (except erythema) or a clinically identifiable, neurologic disorder. All patients who met this definition of vulvodynia, including generalized and localized subsets, were categorized as having vulvodynia.

\section{Analytic strategy}

First, we compared characteristics of patients with vulvodynia and those with other vulvar conditions. Next we compared quality of life of patients with vulvodynia, other vulvar conditions, and those with other skin disorders, based on reports in the literature. ${ }^{3}$ Finally, to determine the independent relationship of patient characteristics to quality of life, we performed logistic regression models of poor skin-related quality of life, which we defined as a Skindex score at or above the median score for the entire sample. Independent variables were those we reasoned might likely affect quality of life, and included: age, marital status, ethnicity, diagnosis of vulvodynia, history of depression, and history of anxiety disorder. Logistic models were used because Skindex scores, like many quality-of-life scores, are nonnormally distributed. Separate models were tested in the entire sample and in the subgroup of patients with vulvodynia. 
All statistical analyses were conducted using software (Stata 9, StataCorp-LP, College Station, TX). Differences between group averages for continuous data were tested for significance using Student $t$ test. For categorical variables, the Chi-squared statistic was calculated.

\section{RESULTS}

\section{Patient characteristics}

Of patients recruited to participate, $280(95 \%)$ responded. The sample consisted of 101 patients (36\%) with vulvodynia, and 179 patients (63\%) with other vulvar conditions. Other diagnoses included lichen sclerosus (11\%), lichen simplex chronicus (11\%), lichen planus $(6 \%)$, vulvovaginal candidiasis $(6 \%)$, vulvar intraepithelial neoplasia $(1 \%)$, and a variety of other less common diagnoses $(28 \%)$.

Characteristics of the two diagnostic groups are shown in Table I. Women with vulvodynia were significantly more likely to describe themselves as "white" than women with other vulvar conditions ( $92 \%$ vs $78 \%, P=.040$ ). The groups were similar, however, in age, health status, duration of vulvar symptoms, and location and overall frequency of symptoms.

With respect to comorbidities, women with vulvodynia were significantly more likely than those with other vulvar conditions to report a history of frequent yeast infections (65\% vs $36 \%$, $P<.001)$, interstitial cystitis ( $11 \%$ vs $3 \%, P=.006)$, and depression ( $47 \%$ vs $28 \%, P=.002$ ). They were somewhat more likely to report a history of anxiety disorder $(21 \% \mathrm{vs} 13 \%, P=$. 074).

\section{Quality of life}

In the symptoms domain, patients with vulvodynia experienced worse quality of life than patients with many dermatologic disorders except eczematous dermatitis (Table II). In the emotions and functioning domain, women with vulvodynia had significantly worse quality of life than patients with many dermatologic disorders, including psoriasis, eczematous dermatitis, and acne vulgaris.

Compared with patients with other vulvar conditions, patients with vulvodynia experienced similar quality of life in the symptoms and emotions domains. In the functioning domain, however, patients with vulvodynia experienced significantly worse quality of life than women with other vulvar conditions.

Table III reports the responses of patients about specific aspects of quality of life. Within the symptoms domain, women with vulvodynia were more likely than those with other vulvar conditions to report that their vulva often or always hurts $(60 \%$ vs $46 \%, P=.027)$. On the other hand, they were less likely to report that their vulva itches $(22 \%$ vs $60 \%, P<.001)$. Thus, although overall symptoms scores in the two groups were similar, the types of symptoms experienced were distinctive.

On the emotions scale, women with vulvodynia were more likely than others in the sample to report that their vulvar condition often or always makes them feel depressed (41\% vs $25 \%$, $P=.006)$, angry ( $44 \%$ vs $26 \%, P=.002)$, or frustrated ( $81 \%$ vs $70 \%, P=.039)$. There was no significant difference between the two groups of vulvar conditions in response to items about shame and embarrassment.

Women with vulvodynia were more likely than others in the sample to report that their vulvar condition affects many aspects of their social and physical functioning. In particular, women with vulvodynia were more likely to report that their condition affects how close they can be with those they love (61\% vs $42 \%, P=.002)$, that it makes showing affection difficult (44\% 
vs $22 \%, P<.001)$, and that it interferes with their sex life $(85 \%$ vs $55 \%, P<.001)$. Women with vulvodynia experienced greater difficulty with work and hobbies as well (36\% vs $24 \%$, $P=.042)$.

Responses to selected quality-of-life items that are not part of Skindex are also shown in Table III. Compared with women with other vulvar conditions, patients with vulvodynia were significantly more likely to report that they often or always: feel lonely $(23 \%$ vs $9 \%, P=.002)$, worry that their vulvar condition will continue ( $84 \%$ vs $67 \%, P=.003)$, or worry that their vulvar condition will affect their ability to have a happy relationship (56\% vs $26 \%, P<.001)$.

\section{Multivariable analyses}

Among women with all vulvar conditions, the independent characteristic most strongly related to poor quality of life was a diagnosis of vulvodynia, which was significantly associated with poor functioning (odds ratio $=1.75,95 \%$ confidence interval $1.01-3.05$ ). The only other characteristic that was independently related was age; younger women were more likely to have poor quality of life in the emotional domain (Table IV).

Among the subgroup of women with vulvodynia, a consistent and strong independent relationship was found between a self-reported history of depression and poor quality of life in all domains. Women with vulvodynia who had a self-reported history of depression were 3 to 5 times more likely to have poor quality of life than women with vulvodynia who had no reported history of depression (Table IV).

\section{DISCUSSION}

We found that patients with vulvodynia, like those with other vulvar conditions, had poor quality of life in all domains measured. In fact, the effects of vulvodynia on social and physical functioning were worse than those of other vulvar conditions. This is consistent with prior studies that have found broad effects on quality of life among women with vulvodynia. ${ }^{4-6}$ Among women with vulvodynia, a self-reported history of depression was the only characteristic independently associated with worse quality of life.

Compared with dermatologic disorders conventionally regarded as affecting well-being (eg, psoriasis), patients with vulvodynia experienced a more severe impact on quality of life. These findings will be useful to clinicians who treat vulvar disorders, because they illustrate the magnitude and type of impact that vulvodynia has on the quality of life of their patients. Such understanding should enhance the clinician's ability to provide supportive care.

Analysis of the responses to individual Skindex items allows for a more nuanced understanding of these results. In the emotions domain, women with vulvodynia reported feeling depressed, angry, and frustrated significantly more often than women with other vulvar conditions. The frustration that women with vulvodynia experience could, in part, stem from their significantly higher concern that their vulvar condition will continue ( $84 \%$ vs $67 \%, P=.003$ ). Likewise, the experience of vulvodynia disproportionately affects the ability of women to form and maintain social relationships. Most women with vulvodynia reported that their condition often or always affects how close they can be with those they love, makes showing affection difficult, and interferes with their sex life. Based on these results, one might postulate that vulvodynia, by impacting a woman's sexual functioning, can interfere with intimacy and result in generalized social dysfunction. This in turn could lead to frustration, anger, and depression.

Recent attention has focused on potential comorbidities with vulvodynia. ${ }^{4}$ As in previous studies, ${ }^{7-9}$ we found that women with vulvodynia were more likely to report a history of frequent vaginal yeast infections than women with other vulvar conditions $(65 \%$ vs $36 \%, P<$. 
001), although some of these women may have been given a misdiagnosis of yeast infections before receiving a diagnosis of vulvodynia. Whether this finding is clinically relevant remains to be elucidated.

Women with vulvodynia were more likely to report a history of other disorders that, like vulvodynia, may operate by a centrally mediated pain-enhancing mechanism. ${ }^{10}$ For example, we confirmed that women with vulvodynia were significantly more likely to report a history of interstitial cystitis (11\% vs $3 \%, P=.006)$, an association that has been documented since the 1990 s. ${ }^{11,12}$ Like Arnold et al, ${ }^{4}$ we found that women with vulvodynia were somewhat more likely to have fibromyalgia ( $7 \%$ vs $3 \%, P=.126$ ) and chronic fatigue syndrome (9\% vs $5 \%, P=.244$ ), although our results did not reach statistical significance. Overlapping mechanisms in pain perception could account for the association between vulvodynia and a variety of other pain syndromes. ${ }^{10,13}$

Finally, as has been shown previously for multiple dermatologic conditions ${ }^{14}$ along with vulvodynia, ${ }^{5}$ depression correlates strongly with reduced quality of life. In our sample, women with vulvodynia were significantly more likely than patients with other vulvar conditions to report a history of depression ( $47 \%$ vs $28 \%, P=.002)$. In addition, women with vulvodynia were significantly more likely than women with other vulvar disorders to report that they often or always feel depressed by their vulvar condition ( $41 \%$ vs $25 \%, P=.006)$. Although the effects on quality of life could cause depression, it is also possible that depression could potentiate the impact of vulvodynia on emotional functioning.

\section{Limitations}

Patients in this study were evaluated at one academic medical center, and the results may not be typical of patients elsewhere. Nonetheless, the sample is large and the response rate was high. In addition, measurement of comorbid conditions such as depression was by self-report, and may not be valid diagnostically. Finally, the study was cross-sectional, and cannot determine time course of illness, or causal features.

\section{Theoretic framework relating vulvodynia to quality of life}

Based on the results of our study, we propose a theoretic framework for the effects of vulvodynia on the quality of life of affected women (Fig 1). We suggest that vulvodynia begins with an as-yet unspecified initiating event that occurs within a particular context. Once initiated, the experience of vulvodynia leads to physical dysfunction, limiting activities related to work, hobbies, and sexual relations. Socially, vulvodynia interferes with a woman's ability to initiate and maintain relationships. Emotionally, women experience frustration, anger, and sadness. These effects should not be viewed as independent from each other, but as interacting. For example, discomfort with sexual intercourse (physical) can impact a woman's ability to develop and maintain intimate relationships (social), which can in turn lead to feelings of loneliness, anger, and frustration (emotional).

\section{CONCLUSIONS}

This study confirms the profound impact that vulvodynia has on quality of life. Further studies are required to refine the theoretic model we propose, to elucidate the nature of the relationship between vulvodynia and depression, and to develop a standard means for assessing quality-oflife outcomes in clinical trials of vulvodynia. Most importantly, the results of this study and future studies should aid clinicians in responding to and caring for patients with vulvodynia. 


\section{Acknowledgments}

Supported an Independent Scientist Award (K02 AR 02203) and a Midcareer Investigator Award (K24-AR052667) to Dr Chren from the National Institute of Arthritis and Musculoskeletal and Skin Diseases, National Institutes of Health.

\section{REFERENCES}

1. Moyal-Barracco M, Lynch PJ. 2003 ISSVD terminology and classification of vulvodynia: a historical perspective. J Reprod Med 2004;49:772-777. [PubMed: 15568398]

2. Harlow BL, Stewart EG. A population-based assessment of chronic unexplained vulvar pain: have we underestimated the prevalence of vulvodynia? J Am Med Womens Assoc 2003;58:82-88. [PubMed: 12744420]

3. Chren MM, Lasek RJ, Flocke SA, Zyzanski SJ. Improved discriminative and evaluative capability of a refined version of Skindex, a quality-of-life instrument for patients with skin diseases. Arch Dermatol 1997;133:1433-1440. [PubMed: 9371029]

4. Arnold LD, Bachmann GA, Rosen R, Kelly S, Rhoads GG. Vulvodynia: characteristics and associations with comorbidities and quality of life. Obstet Gynecol 2006;107:617-624. [PubMed: 16507933]

5. Masheb RM, Wang E, Lozano C, Kerns RD. Prevalence and correlates of depression in treatmentseeking women with vulvodynia. J Obstet Gynaecol 2005;25:786-791. [PubMed: 16368586]

6. Schmidt S, Bauer A, Greif C, Merker A, Elsner P, Strauss B. Vulvar pain: psychological profiles and treatment responses. J Reprod Med 2001;46:377-384. [PubMed: 11354840]

7. Friedrich EG Jr. Vulvar vestibulitis syndrome. J Reprod Med 1987;32:110-114. [PubMed: 3560069]

8. Lynch PJ. Vulvodynia: a syndrome of unexplained vulvar pain, psychologic disability and sexual dysfunction. J Reprod Med 1986;31:773-780. [PubMed: 2430101]

9. Sadownik LA. Clinical profile of vulvodynia patients: a prospective study of 300 patients. J Reprod Med 2000;45:679-684. [PubMed: 10986689]

10. Edwards L. New concepts in vulvodynia. Am J Obstet Gynecol 2003;189:S24-S30. [PubMed: 14532900]

11. Fitzpatrick CC, DeLancey JO, Elkins TE, McGuire EJ. Vulvar vestibulitis and interstitial cystitis: a disorder of urogenital sinus-derived epithelium? Obstet Gynecol 1993;81:860-862. [PubMed: 8469499]

12. McCormack WM. Two urogenital sinus syndromes: interstitial cystitis and focal vulvitis. J Reprod Med 1990;35:873-876. [PubMed: 2231561]

13. Gaitonde P, Rostron J, Longman L, Field EA. Burning mouth syndrome and vulvodynia coexisting in the same patient: a case report. Dent Update 2002;29:75-76. [PubMed: 11928344]

14. Picardi A, Abeni D, Melchi CF, Puddu P, Pasquini P. Psychiatric morbidity in dermatological outpatients: an issue to be recognized. Br J Dermatol 2000;143:983-991. [PubMed: 11069507] 


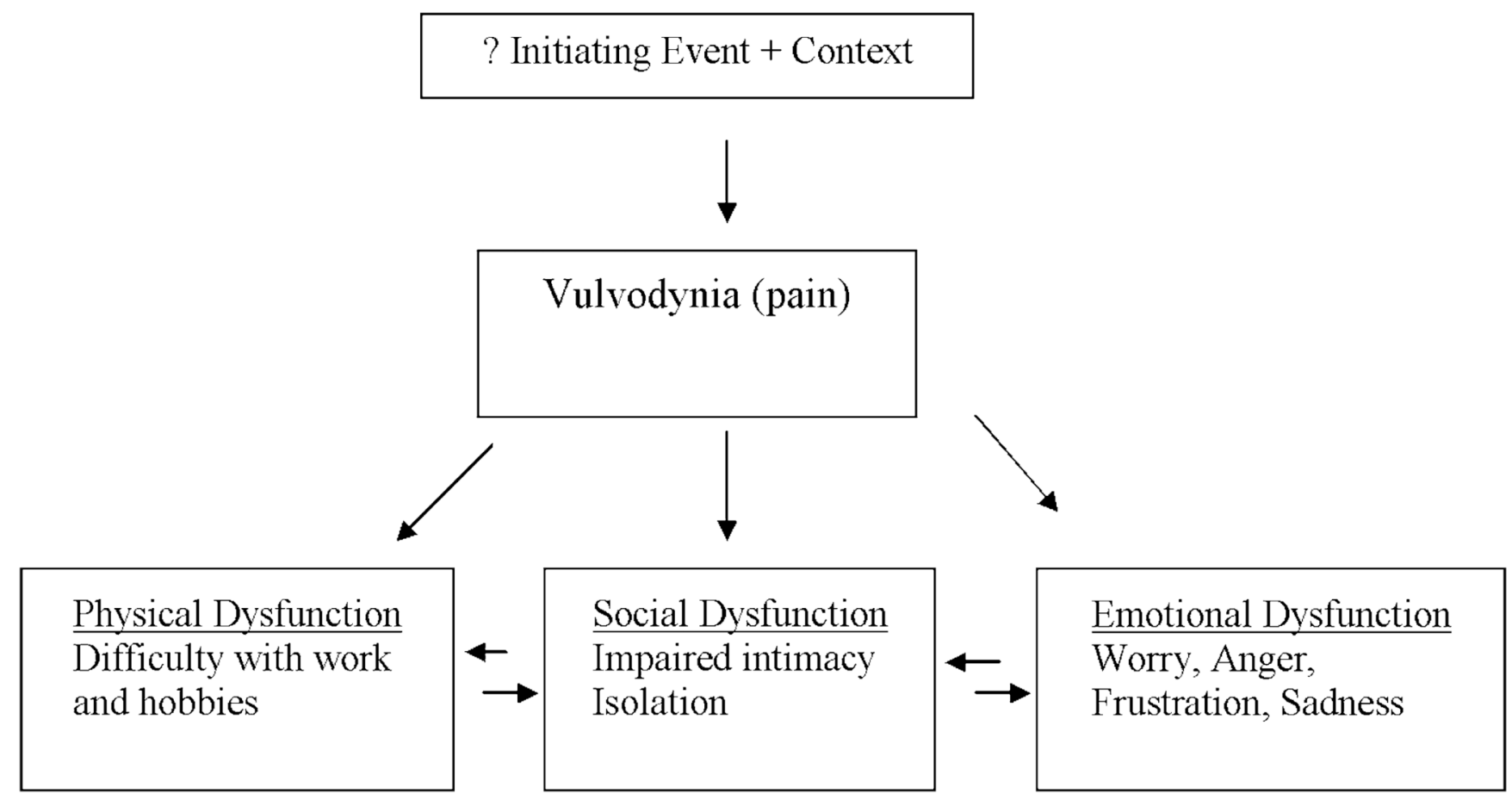

Fig 1.

Framework for effects of vulvodynia on quality of life of affected women. 
Table I

Characteristics of 280 patients seen at a vulvar disorders clinic

\begin{tabular}{|c|c|c|c|}
\hline Demographic characteristics ${ }^{*}$ & Vulvodynia $(n=101)$ & $\begin{array}{l}\text { Other vulvar condition } \\
\qquad(\mathrm{n}=179)\end{array}$ & $P$ value ${ }^{\dagger}$ \\
\hline Age & $42( \pm 16)$ & $45( \pm 17)$ & .149 \\
\hline Marital status & & & .966 \\
\hline Single & $37 \%(37)$ & $39 \%(69)$ & \\
\hline Married & $51 \%(50)$ & $49 \%(87)$ & \\
\hline Other ${ }^{*}$ & $12 \%(12)$ & $12 \%(22)$ & \\
\hline Ethnicity & & & .040 \\
\hline White & $92 \%(80)$ & $78 \%(125)$ & \\
\hline Asian/Pacific Islander & $4 \%(3)$ & $9 \%(15)$ & \\
\hline African American & $0 \%(0)$ & $1 \%(2)$ & \\
\hline Other & $5 \%(4)$ & $12 \%(19)$ & \\
\hline Global health status & & & .257 \\
\hline Poor & $5 \%(5)$ & $3 \%(5)$ & \\
\hline Fair & $15 \%(15)$ & $16 \%(28)$ & \\
\hline Good & $35 \%(34)$ & $38 \%(65)$ & \\
\hline Very good & $26 \%(25)$ & $33 \%(58)$ & \\
\hline Excellent & $19 \%(18)$ & $10 \%(18)$ & \\
\hline \multicolumn{4}{|l|}{ Vulvar symptom characteristics } \\
\hline Duration & $5( \pm 8)$ & $5( \pm 9)$ & .851 \\
\hline \multicolumn{4}{|l|}{ Location } \\
\hline Generalized & $39 \%(36)$ & $45 \%(78)$ & .356 \\
\hline Localized & $61 \%(57)$ & $55 \%(97)$ & \\
\hline \multicolumn{4}{|l|}{ Frequency } \\
\hline Intermittent & $52 \%(49)$ & $51 \%(87)$ & .950 \\
\hline Constant & $48 \%(46)$ & $49 \%(83)$ & \\
\hline \multicolumn{4}{|l|}{ Comorbidities } \\
\hline Frequent urinary infections & $29 \%(28)$ & $22 \%(38)$ & .180 \\
\hline Frequent yeast infections & $65 \%(64)$ & $36 \%(62)$ & .000 \\
\hline Interstitial cystitis & $11 \%(11)$ & $3 \%(5)$ & .006 \\
\hline Fibromyalgia & $7 \%(7)$ & $3 \%(5)$ & .126 \\
\hline Chronic fatigue syndrome & $9 \%(9)$ & $5 \%(3)$ & .244 \\
\hline Irritable bowel syndrome & $25 \%(25)$ & $20 \%(34)$ & .325 \\
\hline Depression & $47 \%(47)$ & $28 \%(49)$ & .002 \\
\hline Anxiety disorder & $21 \%(21)$ & $13 \%(22)$ & .074 \\
\hline
\end{tabular}

$*$ No. missing: age $=1$, marital status $=3$, ethnicity $=32$, comorbidities $=6-18$, health status $=9$, symptom characteristics $=12-15$.

${ }^{\dagger} P$ value refers to comparison of patients with vulvodynia to those with other vulvar conditions.

Fther = widowed, divorced, separated. 
Table II

Mean Skindex scores $( \pm \mathrm{SD})$ of patients with vulvodynia compared to patients with other vulvar conditions and other skin conditions

\begin{tabular}{|c|c|c|c|}
\hline Diagnosis (No. of patients* ) & Symptoms & Emotions & Functioning \\
\hline Vulvodynia $(\mathrm{n}=98)$ & $50( \pm 17)$ & $50( \pm 20)$ & $44( \pm 22)$ \\
\hline $\begin{array}{l}\text { Other vulvar } \\
\text { disorders }(\mathrm{n}=164)\end{array}$ & $48( \pm 22)$ & $46( \pm 24)$ & $34( \pm 24)^{\dagger}$ \\
\hline Psoriasis $(\mathrm{n}=44)$ & $42( \pm 21)^{\dagger}$ & $39( \pm 27)^{\dagger}$ & $23( \pm 27)^{\dagger}$ \\
\hline Eczema $(n=102)$ & $48( \pm 23)$ & $41( \pm 27)^{\dagger}$ & $26( \pm 26)^{\dagger}$ \\
\hline $\begin{array}{l}\text { Acne vulgaris } \\
(\mathrm{n}=63)\end{array}$ & $30( \pm 19)^{\dagger}$ & $41( \pm 25)^{\dagger}$ & $16( \pm 16)^{\dagger}$ \\
\hline $\begin{array}{l}\text { Seborrheic } \\
\text { dermatitis }(n=44)\end{array}$ & $40( \pm 25)^{\dagger}$ & $36( \pm 27)^{\dagger}$ & $23( \pm 25)^{\dagger}$ \\
\hline $\begin{array}{l}\text { Without skin } \\
\text { disease }(\mathrm{n}=107)\end{array}$ & $14( \pm 12)^{\dagger}$ & $9( \pm 13)^{\dagger}$ & $4( \pm 8)^{\dagger}$ \\
\hline
\end{tabular}

* No. of patients with vulvodynia for whom a score was available in all 3 domains.

${ }_{P}^{\dagger}<.05$ when compared with patients with vulvodynia. 
Table III

Comparison of responses with selected quality-of-life items

Those who responded "often" or "always"

Selected Skindex items

Those who responded "often" or "always"

\begin{tabular}{|c|c|c|c|}
\hline Selected Skındex items & Vuivodynia $(n=101)$ & condition $(n=179)$ & \\
\hline \multicolumn{4}{|l|}{ Symptoms } \\
\hline My vulva hurts & $60 \%$ & $46 \%$ & .027 \\
\hline My vulva itches & $22 \%$ & $60 \%$ & .000 \\
\hline \multicolumn{4}{|l|}{ Emotions } \\
\hline My vulvar condition makes me feel depressed & $41 \%$ & $25 \%$ & .006 \\
\hline I am angry about my vulvar condition & $44 \%$ & $26 \%$ & .002 \\
\hline I am frustrated by my vulvar condition & $81 \%$ & $70 \%$ & .039 \\
\hline I am ashamed of my vulvar condition & $24 \%$ & $24 \%$ & .930 \\
\hline I am embarrassed by my vulvar condition & $29 \%$ & $25 \%$ & .439 \\
\hline \multicolumn{4}{|l|}{ Functioning } \\
\hline \multicolumn{4}{|l|}{ My vulvar condition } \\
\hline ....affects my social life & $36 \%$ & $19 \%$ & .002 \\
\hline ... affects how close I can be with those I love & $61 \%$ & $42 \%$ & .002 \\
\hline ...makes showing affection difficult & $44 \%$ & $22 \%$ & .000 \\
\hline ...is a problem for the people I love & $46 \%$ & $28 \%$ & .002 \\
\hline ...interferes with my sex life & $85 \%$ & $55 \%$ & .000 \\
\hline ...makes it hard to work or do hobbies & $36 \%$ & $24 \%$ & .042 \\
\hline ....affects how well I sleep & $19 \%$ & $20 \%$ & .823 \\
\hline \multicolumn{4}{|l|}{ Selected other items } \\
\hline \multicolumn{4}{|l|}{ My vulvar condition } \\
\hline ...makes me feel lonely & $23 \%$ & $9 \%$ & .002 \\
\hline ...makes me feel hopeless & $36 \%$ & $27 \%$ & .103 \\
\hline ...affects my ability to have a happy relationship & $56 \%$ & $26 \%$ & .000 \\
\hline I worry that my vulvar condition will continue & $84 \%$ & $67 \%$ & .003 \\
\hline
\end{tabular}

* No. of missing responses: Skindex items (4-26), other items (14-17).

${ }^{\dagger} P$ value refers to the comparison of patients with vulvodynia to those with other vulvar conditions. 
Table IV

Multivariable model of poor quality of life in women with vulvar conditions and in the subset of women with vulvodynia

\begin{tabular}{|c|c|c|c|c|}
\hline \multirow[b]{2}{*}{ Variable } & \multicolumn{2}{|c|}{$\begin{array}{l}\text { Entire sample with any vulvar disorder } \\
\qquad(\mathrm{n}=164)\end{array}$} & \multicolumn{2}{|c|}{ Vulvodynia alone $(\mathbf{n}=98)$} \\
\hline & OR & $95 \% \mathrm{CI}$ & OR & $95 \% \mathrm{CI}$ \\
\hline \multicolumn{5}{|l|}{ Symptoms score } \\
\hline Increasing age & 0.99 & $0.98-1.01$ & 1.01 & $0.98-1.03$ \\
\hline Married ${ }^{*}$ & 0.78 & $0.46-1.33$ & 0.82 & $0.32-2.09$ \\
\hline White $^{\dagger}$ & 1.53 & $0.74-3.15$ & 3.56 & $0.58-21.97$ \\
\hline Diagnosis of vulvodynia & 1.04 & $0.60-1.81$ & & \\
\hline History of depression & 1.01 & $0.99-1.04$ & 3.13 & $1.20-8.15$ \\
\hline History of anxiety & 1.01 & $0.99-1.03$ & 1.07 & $0.59-1.95$ \\
\hline \multicolumn{5}{|l|}{ Emotions score } \\
\hline Increasing age & 0.98 & $0.96-0.99$ & 0.98 & $0.96-1.01$ \\
\hline Married $^{*}$ & 0.98 & $0.57-1.68$ & 0.69 & $0.27-1.81$ \\
\hline White $^{\dagger}$ & 0.77 & $0.37-1.59$ & 0.56 & $0.08-3.81$ \\
\hline Diagnosis of vulvodynia & 1.50 & $0.86-2.63$ & & \\
\hline History of depression & 1.02 & $0.99-1.04$ & 2.71 & $1.03-7.16$ \\
\hline History of anxiety & 1.01 & $0.98-1.03$ & 1.96 & $0.56-6.86$ \\
\hline \multicolumn{5}{|l|}{ Functioning score } \\
\hline Increasing age & 0.99 & $0.97-1.01$ & 1.01 & $0.98-1.04$ \\
\hline Married ${ }^{*}$ & 1.55 & $0.91-2.66$ & 0.86 & $0.33-2.25$ \\
\hline White ${ }^{\dagger}$ & 0.98 & $0.48-2.01$ & 1.36 & $0.23-8.08$ \\
\hline Diagnosis of vulvodynia & 1.75 & $1.01-3.05$ & & \\
\hline History of depression & 1.03 & $0.99-1.06$ & 4.90 & $1.78-13.52$ \\
\hline History of anxiety & 0.99 & $0.97-1.02$ & 1.13 & $0.32-3.99$ \\
\hline
\end{tabular}

CI, Confidence interval; OR, odds ratio.

In reference to those who are single, divorced, widowed, or separated.

${ }^{\dagger}$ In reference to those of other ethnicities. 